\title{
CMAP Ontology ID
}

National Cancer Institute

\section{Source}

National Cancer Institute. CMAP Ontology ID. NCI Thesaurus. Code C41082.

An object providing entry to the CMAP gene ontology. 